International Journal of Pure and Applied Mathematics

Volume 96 No. 1 2014, 59-78

ISSN: 1311-8080 (printed version); ISSN: 1314-3395 (on-line version)

url: http://www.ijpam.eu

doi: http://dx.doi.org/10.12732/ijpam.v96i1.6

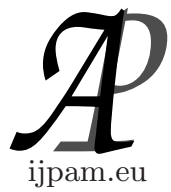

\title{
EXPONENTIAL SYNCHRONIZATION OF MASTER-SLAVE NEURAL NETWORKS WITH MIXED TIME-VARYING DELAYS VIA HYBRID INTERMITTENT FEEDBACK CONTROL
}

\author{
T. Botmart \\ Department of Mathematics \\ Srinakharinwirot University \\ Bangkok, 10110, THAILAND
}

\begin{abstract}
In this paper, we investigate the problem of exponential synchronization for master-slave neural networks with mixed time-varying delays via hybrid intermittent feedback control. The constraint on the derivative of the time-varying delay is not required which allows the time-delay to be a fast timevarying function. Based on the construction of improved Lyapunov-Krasovskii functionals is combined with Leibniz-Newtons formula and the technique of dealing with some integral terms. New delay-dependent sufficient conditions for the exponential synchronization of the error systems with memoryless hybrid feedback control are first established in terms of LMIs without introducing any free-weighting matrices. The designed controller ensures that the synchronization of the error systems are proposed via hybrid intermittent feedback control. Numerical simulations are presented to illustrate the effectiveness of these synchronization criteria.
\end{abstract}

AMS Subject Classification: 34D06, 92B20, 93B52

Key Words: master-slave neural networks, exponential synchronization, mixed time-varying delays, intermittent feedback control

Received: May 15, 2014

(c) 2014 Academic Publications, Ltd. url: www.acadpubl.eu 


\section{Introduction}

In the past decade, synchronization in neural networks (NNs), such as cellular NNs, hopfield NNs and bi-directional associative memory networks, has received a great deal of interest among scientists in a variety of areas, such as signal processing, pattern recognition, static image processing, associative memory, content-addressable memory and combinatorial optimization $[1,2,3,4]$.

In performing a periodicity or stability analysis of a neural network, the conditions to be imposed on the neural network are determined by the characteristics of various activation functions and network parameters. When neural networks are created for problem solving, it is desirable for their activation functions are not too restrictive. As a result, there has been considerable research work on the stability of neural networks with various activation functions and more general conditions [5,6]. The first concept of chaos synchronization is making two chaotic systems oscillate in a synchronized manner was introduced by [2] and many different methods have been applied theoretically and experimentally to synchronize chaotic systems, for example active control [7], adaptive control [7], time-delay feedback control [?, 8] and intermittent control [9], etc. Moreover, neural networks with distributed delays have been extensively discussed $[8,10,11,12]$. In [12], a neural circuit has been designed with distributed delays, which solves the general problem of recognized patterns in a time-dependent signal. The master-slave synchronization problem has been investigated for neural networks with discrete and distributed time-varying delays in [10], based on the drive-response concept, LMI approach and the Lyapunov stability theorem, several delay-dependent feedback controllers were derived to achieve the exponential synchronization of the chaotic neural networks.

Intermittent control is one of discontinuous control and has a nonzero control width. It is an engineering approach that has been widely used in engineering fields, such as manufacturing, air-quality control,transportation and communication in practice. However, results using intermittent control to study exponential synchronization are few. In fact, to use synchronization of NNs in some intervals only may prove to be more cost effective than using synchronization of NNs at all times. Another reason could be that the intermittent control scheme might be useful in communications, where synchronization of NNs between transmitter and receiver can be used as means of transmitting information [13]. In recent years, several synchronization criteria for neural networks with or without time delays via intermittent control have been presented, see $[14,15,16,17,18]$. In [16], the problem of synchronization for a class of CohenGrossberg neural networks with time delays under periodical in- 
termittent control has been investigated. The quasi-synchronization problem has been investigated for chaotic systems with parameter mismatch by means of periodically intermittent control and design a general periodically-intermittent controller for chaotic systems in [15]. To the best of the author's knowledge, the problem of exponential synchronization for master-slave neural networks with mixed time-varying delays in state and control have not been fully investigated yet and remains open.

This paper, inspired by the above discussions, we shall investigate the problem of exponential synchronization for master-slave neural networks with mixed time-varying delays, which is composed of discrete interval time-varying delay and distributed time-varying delay. The designed controller ensures that the synchronization of delayed master-slave neural networks are proposed via hybrid intermittent feedback control.There are various activation functions which are considered in the system and the restriction on differentiability of interval time-varying delays is removed. Based on the construction of improved Lyapunov-Krasovskii functional is combined with Leibniz-Newton formula and the technique of dealing with some integral terms. New synchronization criteria are derived in terms of LMIs which can be solved efficiently by standard convex optimization algorithms. A numerical example is also given to illustrate the effectiveness of the proposed method.

The rest of this paper is organized as follows. In Section 2, we give notations, definition, propositions and lemma for using in the proof of the main results. In Section 3, synchronization stability in master-slave neural networks with mixed time-varying delays via hybrid intermittent feedback control are investigated. Numerical examples illustrated the obtained results are given in Section 4. The paper ends with conclusions in Section 5.

\section{Preliminaries}

The following notation will be used in this paper: $R^{+}$denotes the set of all real non-negative numbers; $R^{n}$ denotes the $n$-dimensional space and the vector norm $\|.\| ; M^{n \times r}$ denotes the space of all matrices of $(n \times r)$-dimensions.

$A^{T}$ denotes the transpose of matrix $A ; A$ is symmetric if $A=A^{T} ; I$ denotes the identity matrix; $\lambda(A)$ denotes the set of all eigenvalues of $A ; \lambda_{\max }(A)=$ $\max \{\operatorname{Re} \lambda ; \lambda \in \lambda(A)\}$.

Matrix $A$ is called semi-positive definite $(A \geq 0)$ if $\langle A x, x\rangle \geq 0$, for all $x \in R^{n} ; A$ is positive definite $(A>0)$ if $\langle A x, x\rangle>0$ for all $x \neq 0 ; A>B$ means $A-B>0$. The symmetric term in a matrix is denoted by $*$. 
In this paper, the master-slave cellular neural networks (MSCNNs) with mixed time-varying delays are described as follows:

$$
\begin{aligned}
\dot{x}(t)= & -A x(t)+C \tilde{f}(x(t))+D \tilde{g}\left(x\left(t-h_{1}(t)\right)\right) \\
& +E \int_{t-k_{1}(t)}^{t} \tilde{h}(x(s)) d s+I(t), \\
x(t)= & \phi_{1}(t), \quad t \in[-d, 0], \\
\dot{y}(t)= & -A y(t)+C \tilde{f}(y(t))+D \tilde{g}\left(y\left(t-h_{1}(t)\right)\right) \\
& +E \int_{t-k_{1}(t)}^{t} \tilde{h}(y(s)) d s+I(t)+\mathcal{U}(t), \\
y(t)= & \phi_{2}(t), \quad t \in[-d, 0],
\end{aligned}
$$

where $x(t)=\left[x_{1}(t), x_{2}(t), \ldots, x_{n}(t)\right] \in R^{n}, y(t)=\left[y_{1}(t), y_{2}(t), \ldots, y_{n}(t)\right] \in R^{n}$ are the master systems state vector and the slave systems state vector of the neural networks, respectively. $n$ is the number of neural, and

$$
\begin{aligned}
\tilde{f}(x(t)) & =\left[\tilde{f}_{1}\left(x_{1}(t)\right), \tilde{f}_{2}\left(x_{2}(t)\right), \ldots, \tilde{f}_{n}\left(x_{n}(t)\right)\right]^{T}, \\
\tilde{g}(x(t)) & =\left[\tilde{g}_{1}\left(x_{1}(t)\right), \tilde{g}_{2}\left(x_{2}(t)\right), \ldots, \tilde{g}_{n}\left(x_{n}(t)\right)\right]^{T}, \\
\tilde{h}(x(t)) & =\left[\tilde{h}_{1}\left(x_{1}(t)\right), \tilde{h}_{2}\left(x_{2}(t)\right), \ldots, \tilde{h}_{n}\left(x_{n}(t)\right)\right]^{T},
\end{aligned}
$$

are the activation functions, $A=\operatorname{diag}\left(\overline{a_{1}}, \overline{a_{2}}, \ldots, \overline{a_{n}}\right), \overline{a_{i}}>0$ represents the self-feedback term and $C, D, E$ denote the connection weights, the discretely delayed connection weights and the distributively delayed connection weight, respectively.

The synchronization error $e(t)$ is the form $e(t)=y(t)-x(t)$. Therefore, the cellular neural networks with mixed time-varying delays of synchronization error between the master-slave systems given in (1) and (2) can be described by

$$
\begin{aligned}
\dot{e}(t)= & -A e(t)+C f(e(t))+D g\left(e\left(t-h_{1}(t)\right)\right) \\
& +E \int_{t-k_{1}(t)}^{t} h(e(s)) d s+\mathcal{U}(t), \\
e(t)= & \phi_{2}(t)-\phi_{1}(t)=\phi(t), \quad t \in[-d, 0],
\end{aligned}
$$

where $f(e(t))=\tilde{f}(e(t)+x(t))-\tilde{f}(x(t)), g\left(e\left(t-h_{1}(t)\right)\right)=\tilde{g}\left(e\left(t-h_{1}(t)\right)+x(t-\right.$ $\left.\left.h_{1}(t)\right)\right)-\tilde{g}\left(x\left(t-h_{1}(t)\right)\right), \int_{t-k_{1}(t)}^{t} h(e(s)) d s=\int_{t-k_{1}(t)}^{t} h(e(s)+x(s))-h(x(s)) d s$. The state hybrid feedback controller $\mathcal{U}(t)$ satisfying :

$$
\mathcal{U}(t)= \begin{cases}B_{1} u(t)+B_{2} u\left(t-h_{2}(t)\right) & \\ +B_{3} \int_{t-k_{2}(t)}^{t} u(s) d s, & n \omega \leq t \leq n \omega+\delta, \\ 0, & n \omega+\delta<t \leq(n+1) \omega .\end{cases}
$$


where $u(t)=K e(t)$ and $K$ is a constant matrix control gain, $\omega>0$ is the control period and $\delta>0$ is called the control width (control duration) and $n$ is a non-negative integer. In this paper, our goal is to design suitable $K$ such that system (2) synchronizes with system (1). Then, substituting it into (3), it is easy to get the following:

$$
\begin{aligned}
\dot{e}(t)= & -A e(t)+C f(e(t))+D g\left(e\left(t-h_{1}(t)\right)\right)+E \int_{t-k_{1}(t)}^{t} h(e(s)) d s \\
& +B_{1} K e(t)+B_{2} K e\left(t-h_{2}(t)\right)+B_{3} K \int_{t-k_{2}(t)}^{t} e(s) d s, \\
& n \omega \leq t \leq n \omega+\delta, \\
\dot{e}(t)= & -A e(t)+C f(e(t))+D g\left(e\left(t-h_{1}(t)\right)\right)+E \int_{t-k_{1}(t)}^{t} h(e(s)) d s \\
& n \omega+\delta<t \leq(n+1) \omega, \\
e(t)= & \phi_{2}(t)-\phi_{1}(t)=\phi(t), \quad t \in[-d, 0] .
\end{aligned}
$$

Throughout this paper, we consider various activation functions and the activation functions $\tilde{f}(),. \tilde{g}($.$) and \tilde{h}($.$) satisfy the following assumption:$

(A1) The activation functions $\tilde{f}(),. \tilde{g}($.$) and \tilde{h}($.$) satisfy Lipschitzian with$ the Lipschitz constants $\hat{f}_{i}, \hat{g}_{i}>0$ and $\hat{h}_{i}>0$ :

$$
\begin{aligned}
\left|\tilde{f}_{i}\left(\xi_{1}\right)-\tilde{f}_{i}\left(\xi_{2}\right)\right| \leq \hat{f}_{i}\left|\xi_{1}-\xi_{2}\right|, & i=1,2, \ldots, n, \forall \xi_{1}, \xi_{2} \in R, \\
\left|\tilde{g}_{i}\left(\xi_{1}\right)-\tilde{g}_{i}\left(\xi_{2}\right)\right| \leq \hat{g}_{i}\left|\xi_{1}-\xi_{2}\right|, & i=1,2, \ldots, n, \forall \xi_{1}, \xi_{2} \in R, \\
\left|\tilde{h}_{i}\left(\xi_{1}\right)-\tilde{h}_{i}\left(\xi_{2}\right)\right| \leq \hat{h}_{i}\left|\xi_{1}-\xi_{2}\right|, & i=1,2, \ldots, n, \forall \xi_{1}, \xi_{2} \in R,
\end{aligned}
$$

and we denote

$$
\begin{aligned}
& F=\operatorname{diag}\left\{\hat{f}_{i}, \quad i=1,2, \ldots, n\right\}, \\
& G=\operatorname{diag}\left\{\hat{g}_{i}, \quad i=1,2, \ldots, n\right\}, \\
& H=\operatorname{diag}\left\{\hat{h}_{i}, \quad i=1,2, \ldots, n\right\} .
\end{aligned}
$$

The time-varying delay functions $h_{i}(t), k_{i}(t), i=1,2$ satisfy the condition

$$
\begin{aligned}
& 0 \leq h_{1 m} \leq h_{1}(t) \leq h_{1 M}, \quad 0 \leq h_{2}(t) \leq h_{2}, \\
& 0 \leq k_{1}(t) \leq k_{1}, \quad 0 \leq k_{2}(t) \leq k_{2} .
\end{aligned}
$$

It is worth noting that the time delay is assumed to be a continuous function belonging to a given interval, which means that the lower and upper bounds for the time-varying delay are available, but the delay function is bounded 
but not restricted to being zero. The initial functions $\phi(t) \in C^{1}\left([-d, 0], R^{n}\right)$, $d=\max \left\{h_{1 M}, h_{2}, k_{1}, k_{2}\right\}$ with the norm

$$
\|\phi\|=\sup _{t \in[-d, 0]} \sqrt{\|\phi(t)\|^{2}+\|\dot{\phi}(t)\|^{2}} .
$$

Definition 1. Given $\alpha>0$. The zero solution of system (5) with $u(t)=$ $K e(t)$ is $\alpha$ - stable if there exist a positive number $N>0$ such that every solution $e(t, \phi)$ satisfies the following condition:

$$
\|e(t, \phi)\| \leq N e^{-\alpha t}\|\phi\|, \quad \forall t \geq 0 .
$$

We introduce the following technical well-known lemma, which will be used in the proof of our results.

Lemma 1. [19] (Cauchy inequality) For any symmetric positive definite matrix $N \in M^{n \times n}$ and $x, y \in R^{n}$ we have

$$
\pm 2 x^{T} y \leq x^{T} N x+y^{T} N^{-1} y .
$$

Lemma 2. [19] For any symmetric positive definite matrix $M>0$, scalar $\gamma>0$ and vector function $\omega:[0, \gamma] \rightarrow R^{n}$ such that the integrations concerned are well defined, the following inequality holds

$$
\left(\int_{0}^{\gamma} \omega(s) d s\right)^{T} M\left(\int_{0}^{\gamma} \omega(s) d s\right) \leq \gamma\left(\int_{0}^{\gamma} \omega^{T}(s) M \omega(s) d s\right) .
$$

Lemma 3. [19] (Schur complement lemma). Given constant symmetric matrices $X, Y, Z$ with appropriate dimensions satisfying $X=X^{T}, Y=Y^{T}>0$. Then $X+Z^{T} Y^{-1} Z<0$ if and only if

$$
\left(\begin{array}{cc}
X & Z^{T} \\
Z & -Y
\end{array}\right)<0 \quad \text { or } \quad\left(\begin{array}{cc}
-Y & Z \\
Z^{T} & X
\end{array}\right)<0
$$

\section{Main Results}

Let us set

$$
\begin{aligned}
\Upsilon= & e^{-2 \alpha h_{1 M}}, \lambda_{1}=\lambda_{\min }\left(P^{-1}\right), \\
\lambda_{2}= & \lambda_{\max }\left(P^{-1}\right)+\left(h_{1 m}+h_{1 M}\right) \lambda_{\max }\left(P^{-1} Q P^{-1}\right) \\
& +\left(h_{1 m}^{3}+h_{1 M}^{3}\right) \lambda_{\max }\left(P^{-1} R P^{-1}\right)+\delta^{3} \lambda_{\max }\left(P^{-1} U P^{-1}\right) \\
& +h_{2}^{3} \lambda_{\max }\left(P^{-1} Y^{T} S_{1}^{-1} Y P^{-1}\right)+k_{1}^{2} \lambda_{\max }\left(H U_{3}^{-1} H\right) \\
& +k_{2}^{2} \lambda_{\max }\left(P^{-1} Y^{T} S_{2}^{-1} Y P^{-1}\right) .
\end{aligned}
$$


Theorem 2. For some given scalars $0<\alpha<\varepsilon$, the error system (5) with time-varying delay satisfying (7) are exponentially stable if there exist symmetric positive definite matrices $P, Q, R, U, S_{1}, S_{2}$, diagonal matrices $U_{i}, \quad i=1,2,3$ and a matrix $Y$ with appropriately dimensioned such that the following $L M I$ holds:

$$
\begin{aligned}
& \Gamma_{1}=\Gamma_{1}-\left[\begin{array}{lllll}
0 & 0 & -I & I & 0
\end{array}\right]^{T} \Upsilon U\left[\begin{array}{lllll}
0 & 0 & -I & I & 0
\end{array}\right]<0, \\
& \Gamma_{2}=\Gamma_{1}-\left[\begin{array}{lllll}
0 & 0 & 0 & I & -I
\end{array}\right]^{T} \Upsilon U\left[\begin{array}{lllll}
0 & 0 & 0 & I & -I
\end{array}\right]<0, \\
& \Gamma_{3}=\left[\begin{array}{cccc}
\Pi_{11} & 2 P F^{T} & P H^{T} & 2 Y \\
* & -2 U_{1} & 0 & 0 \\
* & * & -U_{3} & 0 \\
* & * & * & -2 e^{-2 \alpha h_{2}} S_{1}
\end{array}\right]<0, \\
& \Gamma_{4}=\left[\begin{array}{cc}
-0.1 P & h_{2}^{2} Y^{T} \\
* & -h_{2}^{2} S_{1}
\end{array}\right]<0, \\
& \Gamma_{5}=\left[\begin{array}{cc}
-0.1 e^{-2 \alpha h_{1 M}} U & 2 P G^{T} \\
* & -2 U_{2}
\end{array}\right]<0, \\
& \Gamma_{6}=\Gamma_{2}-\left[\begin{array}{lllll}
0 & 0 & -I & I & 0
\end{array}\right]^{T} \Upsilon U\left[\begin{array}{lllll}
0 & 0 & -I & I & 0
\end{array}\right]<0, \\
& \Gamma_{7}=\Gamma_{2}-\left[\begin{array}{lllll}
0 & 0 & 0 & I & -I
\end{array}\right]^{T} \Upsilon U\left[\begin{array}{lllll}
0 & 0 & 0 & I & -I
\end{array}\right]<0, \\
& \Gamma_{8}=\left[\begin{array}{ccc}
\Pi_{11} & 2 P F^{T} & k_{1} P H^{T} \\
* & -2 U_{1} & 0 \\
* & * & -k_{1} U_{3}
\end{array}\right]<0, \\
& -\alpha \delta+(\varepsilon-\alpha)(\omega-\delta)<0, \\
& \Gamma_{1}=\left[\begin{array}{ccccc}
\Gamma_{111} & \Gamma_{112} & \Gamma_{13} & 0 & \Gamma_{15} \\
* & \Gamma_{122} & 0 & 0 & 0 \\
* & * & \Gamma_{33} & \Gamma_{34} & 0 \\
* & * & * & \Gamma_{44} & \Gamma_{45} \\
* & * & * & * & \Gamma_{55}
\end{array}\right] \\
& \Gamma_{2}=\left[\begin{array}{ccccc}
\Gamma_{211} & \Gamma_{212} & \Gamma_{13} & 0 & \Gamma_{15} \\
* & \Gamma_{222} & 0 & 0 & 0 \\
* & * & \Gamma_{33} & \Gamma_{34} & 0 \\
* & * & * & \Gamma_{44} & \Gamma_{45} \\
* & * & * & * & \Gamma_{55}
\end{array}\right],
\end{aligned}
$$


where

$$
\begin{aligned}
\Pi_{11}= & -0.1\left(e^{-2 \alpha h_{1 m}}+e^{-2 \alpha h_{1 M}}\right) R \\
\Gamma_{111}= & {[-A+\alpha I] P+P[-A+\alpha I]^{T}-B_{1} Y-Y^{T} B_{1}^{T}+2 Q } \\
& +C^{T} U_{1} C+D^{T} U_{2} D+2 k_{1} e^{2 \alpha k_{1}} E^{T} U_{3} E+3 e^{2 \alpha h_{2}} B_{2}^{T} S_{1} B_{2} \\
& +2 k_{2} e^{2 \alpha k_{2}} B_{3}^{T} S_{2} B_{3}-0.9 e^{-2 \alpha h_{1 m}} R-0.9 e^{-2 \alpha h_{1 M}} R, \\
\Gamma_{211}= & -A+\alpha I] P+P[-A+\alpha I]^{T}+2 Q-2 \varepsilon P+C^{T} U_{1} C \\
& +D^{T} U_{2} D+2 k_{1} e^{2 \alpha k_{1}} E^{T} U_{3} E-0.9 e^{-2 \alpha h_{1 m}} R-0.9 e^{-2 \alpha h_{1 M}} R, \\
\Gamma_{112}= & -P A^{T}-Y^{T} B^{T}, \quad \Gamma_{212}=-P A^{T}, \quad \Gamma_{13}=e^{-2 \alpha h_{1 m}} R, \\
\Gamma_{15}= & e^{-2 \alpha h_{1 M}} R, \\
\Gamma_{122}= & h_{1 m}^{2} R+h_{1 M}^{2} R+\delta^{2} U-1.9 P+C^{T} U_{1} C+D^{T} U_{2} D \\
& +2 k_{1} e^{2 \alpha k_{1}} E^{T} U_{3} E+3 e^{2 \alpha h_{2}} B_{2}^{T} S_{1} B_{2} \\
2 & k_{2} e^{2 \alpha k_{2}} B_{3}^{T} S_{2} B_{3}, \\
\Gamma_{222}= & h_{1 m}^{2} R+h_{1 M}^{2} R+\delta^{2} U-2 P+C^{T} U_{1} C+D^{T} U_{2} D \\
& +2 k_{1} e^{2 \alpha k_{1}} E^{T} U_{3} E, \\
\Gamma_{33}= & -e^{-2 \alpha h_{1 m}} Q-e^{-2 \alpha h_{1 m}} R-e^{-2 \alpha h_{1 M}} U, \\
\Gamma_{34}= & e^{-2 \alpha h_{1 M}} U_{4}, \Gamma_{44}=-1.9 e^{-2 \alpha h_{1 M}} U, \Gamma_{45}=e^{-2 \alpha h_{1 M}} U, \\
\Gamma_{55}= & -e^{-2 \alpha h_{1 M}} Q-e^{-2 \alpha h_{1 M}} R-e^{-2 \alpha h_{1 M}} U, \\
= &
\end{aligned}
$$

then the error system (5) have exponential synchronization. Moreover, the feedback control is

$$
\mathcal{U}(t)=\left\{\begin{array}{l}
-B_{1} Y P^{-1} e(t)-B_{2} Y P^{-1} e\left(t-h_{2}(t)\right) \\
-B_{3} Y P^{-1} \int_{t-k_{2}(t)}^{t} e(s) d s, \quad n \omega \leq t \leq n \omega+\delta \\
0, \quad n \omega+\delta<t \leq(n+1) \omega
\end{array}\right.
$$

Proof. Case I: for $n \omega \leq t \leq n \omega+\delta$, let $W=P^{-1}, z(t)=W e(t)$. Using the feedback control (17) we consider the following Lyapunov-Krasovskii functional

$$
V(e(t))=\sum_{i=1}^{9} V_{i}
$$

where

$$
V_{1}=e^{T}(t) W e(t)
$$




$$
\begin{aligned}
V_{2} & =\int_{t-h_{1 m}}^{t} e^{2 \alpha(s-t)} e^{T}(s) W Q W e(s) d s \\
V_{3} & =\int_{t-h_{1 M}}^{t} e^{2 \alpha(s-t)} e^{T}(s) W Q W e(s) d s \\
V_{4} & =h_{1 m} \int_{-h_{1 m}}^{0} \int_{t+s}^{t} e^{2 \alpha(\theta-t)} \dot{e}^{T}(\theta) W R W \dot{e}(\theta) d \theta d s \\
V_{5}= & h_{1 M} \int_{-h_{1 M}}^{0} \int_{t+s}^{t} e^{2 \alpha(\theta-t)} \dot{e}^{T}(\theta) W R W \dot{e}(\theta) d \theta d s \\
V_{6}= & \delta \int_{-h_{1 M}}^{-h_{1 m}} \int_{t+s}^{t} e^{2 \alpha(\theta-t)} \dot{e}^{T}(\theta) W U W \dot{e}(\theta) d \theta d s \\
V_{7}= & \int_{-k_{1}}^{0} \int_{t+s}^{t} e^{2 \alpha(\theta-t)} h^{T}(e(\theta)) U_{3}^{-1} h(e(\theta)) d \theta d s . \\
V_{8}= & h_{2} \int_{h_{2}}^{0} \int_{t+s}^{t} e^{2 \alpha(\theta-t)} \dot{u}^{T}(\theta) S_{1}^{-1} \dot{u}(\theta) d \theta d s \\
& V_{9}=\int_{-k_{2}}^{0} \int_{t+s}^{t} e^{2 \alpha(\theta-t)} u^{T}(\theta) S_{2}^{-1} u(\theta) d \theta d s .
\end{aligned}
$$

It easy to check that

$$
\lambda_{1}\|e(t)\|^{2} \leq V(t, e(t)) \leq \lambda_{2}\left\|e_{t}(t)\right\|^{2}, \quad \forall t \geq 0
$$

Taking the derivative of $V(t, e(t))$ along the solution of system (5) we have

$$
\begin{aligned}
\dot{V}_{1}= & z^{T}(t)\left[-A P-P A^{T}-2 B_{1} Y\right] z(t)+2 z^{T}(t) C f(e(t)) \\
& +2 z^{T}(t) D g\left(e\left(t-h_{1}(t)\right)\right)+2 z^{T}(t) E \int_{t-k_{1}(t)}^{t} h(e(s)) d s \\
& +2 z^{T}(t) B_{2} u\left(t-h_{2}(t)\right)+2 z^{T}(t) B_{3} \int_{t-k_{2}(t)}^{t} u(s) d s, \\
\dot{V}_{2}= & z^{T}(t) Q z(t)-e^{-2 \alpha h_{1 m}} z^{T}\left(t-h_{1 m}\right) Q z\left(t-h_{1 m}\right)-2 \alpha V_{2}, \\
\dot{V}_{3}= & z^{T}(t) Q z(t)-e^{-2 \alpha h_{1 M}} z^{T}\left(t-h_{1 M}\right) Q z\left(t-h_{1 M}\right)-2 \alpha V_{3}, \\
\dot{V}_{4} \leq & h_{1 m}^{2} \dot{z}^{T}(t) R \dot{z}(t)-h_{1 m} e^{-2 \alpha h_{1 m}} \int_{t-h_{1 m}}^{t} \dot{z}^{T}(s) R \dot{z}(s) d s-2 \alpha V_{4}, \\
\dot{V}_{5} \leq & h_{1 M}^{2} \dot{z}^{T}(t) R \dot{z}(t)-h_{1 M} e^{-2 \alpha h_{1 M}} \int_{t-h_{1 M}}^{t} \dot{z}^{T}(s) R \dot{z}(s) d s-2 \alpha V_{5},
\end{aligned}
$$




$$
\begin{aligned}
\dot{V}_{6} \leq & \delta^{2} \dot{z}^{T}(t) U \dot{z}(t)-\delta e^{-2 \alpha h_{1 M}} \int_{t-h_{1 M}}^{t-h_{1 m}} \dot{z}^{T}(s) R \dot{z}(s) d s-2 \alpha V_{6}, \\
\dot{V}_{7} \leq & k_{1} h^{T}(e(t)) U_{3}^{-1} h(e(t))-e^{-2 \alpha k_{1}} \int_{t-k_{1}}^{t} h^{T}(e(s)) U_{3}^{-1} h(e(s)) d s \\
& -2 \alpha V_{7}, \\
\dot{V}_{8} \leq & h_{2}^{2} \dot{u}^{T}(t) S_{1}^{-1} \dot{u}(t)-h_{2} e^{-2 \alpha h_{2}} \int_{t-h_{2}}^{t} \dot{u}^{T}(s) S_{1}^{-1} \dot{u}(s) d s-2 \alpha V_{8}, \\
\dot{V}_{9} \leq & k_{2}^{2} u^{T}(t) S_{2}^{-1} u(t)-k_{2} e^{-2 \alpha k_{2}} \int_{t-k_{2}}^{t} u^{T}(s) S_{2}^{-1} u(s) d s-2 \alpha V_{9} .
\end{aligned}
$$

For assumption $A 1$, we can obtain the following three inequalities:

$$
\begin{aligned}
\left|f_{i}\left(e_{i}(t)\right)\right| & \leq \hat{f}_{i}\left|e_{i}(t)+x_{i}(t)-x_{i}(t)\right|=\hat{f}_{i}\left|e_{i}(t)\right| \\
\left|g_{i}\left(e_{i}(t)\right)\right| & \leq \hat{g}_{i}\left|e_{i}(t)+x_{i}(t)-x_{i}(t)\right|=\hat{g}_{i}\left|e_{i}(t)\right| \\
\left|h_{i}\left(e_{i}(t)\right)\right| & \leq \hat{h}_{i}\left|e_{i}(t)+x_{i}(t)-x_{i}(t)\right|=\hat{h}_{i}\left|e_{i}(t)\right|
\end{aligned}
$$

Applying Lemma (1) and Lemma (2) and since the matrices $U_{i}, i=1,2,3$ are diagonal, we have

$$
\begin{aligned}
2 z^{T}(t) C f(e(t)) \leq & z^{T}(t) C^{T} U_{1} C z(t)+z^{T}(t) P F^{T} U_{1}^{-1} F P z(t) \\
2 z^{T}(t) D g\left(e\left(t-h_{1}(t)\right)\right) \leq & z^{T}(t) D^{T} U_{2} D z(t) \\
& +z^{T}\left(t-h_{1}(t)\right) P G^{T} U_{2}^{-1} G P z\left(t-h_{1}(t)\right), \\
k_{1} h^{T}(e(t)) U_{3}^{-1} h(e(t)) \leq & k_{1} z^{T}(t) P H^{T} U_{3}^{-1} H P z(t), \\
2 z^{T}(t) E \int_{t-k_{1}(t)}^{t} h(e(s)) d s \leq & 2 k_{1} e^{2 \alpha k_{1}} z^{T}(t) E^{T} U_{3} E z(t) \\
& +\frac{e^{-2 \alpha k_{1}}}{2} \int_{t-k_{1}(t)}^{t} h^{T}(e(s)) U_{3}^{-1} h(e(s)) d s, \\
2 z^{T}(t) B_{2} u\left(t-h_{2}(t)\right) \leq & 3 e^{2 \alpha h_{2}} z^{T}(t) B_{2}^{T} S_{1} B_{2} z(t) \\
& +\frac{e^{-2 \alpha h_{2}}}{3} u^{T}\left(t-h_{2}(t)\right) S_{1}^{-1} u\left(t-h_{2}(t)\right), \\
\int_{t-k_{2}(t)}^{t} u(s) d s \leq & 2 k_{2} e^{2 \alpha k_{2}} z^{T}(t) B_{3}^{T} S_{2} B_{3} z(t) \\
2 z^{T}(t) B_{3} \int_{t-k_{2}(t)} u^{T}(s) S_{2}^{-1} u(s) d s, & +\frac{e^{-2 \alpha k_{2}}}{2} \int^{t} h_{2}^{2} \dot{z}^{T}(t) Y^{T} S_{1}^{-1} \dot{z}_{(t) .}
\end{aligned}
$$


and the Leibniz-Newton formula gives

$$
\begin{aligned}
& -h_{2} e^{-2 \alpha h_{2}} \int_{t-h_{2}}^{t} \dot{u}^{T}(s) S_{1}^{-1} \dot{u}(s) d s \\
& \leq 2 e^{-2 \alpha h_{2}} z^{T}(t) Y^{T} S_{1}^{-1} Y z(t)+\frac{e^{-2 \alpha h_{2}}}{3} u^{T}\left(t-h_{2}(t)\right) S_{1}^{-1} u\left(t-h_{2}(t)\right) \\
& -e^{-2 \alpha h_{2}} u^{T}\left(t-h_{2}(t)\right) S_{1}^{-1} u\left(t-h_{2}(t)\right) .
\end{aligned}
$$

Applying Lemma 2 and the Leibniz-Newton formula, we have

$$
\begin{aligned}
& -h_{1 m} e^{-2 \alpha h_{1 m}} \int_{t-h_{1 m}}^{t} \dot{z}^{T}(s) R \dot{z}(s) d s \leq-e^{-2 \alpha h_{1 m}}\left[z^{T}(t) R z(t)\right. \\
& -2 z^{T}(t) R z\left(t-h_{1 m}\right) \\
& \left.+z^{T}\left(t-h_{1 m}\right) R z\left(t-h_{1 m}\right)\right] \text {, } \\
& -h_{1 M} e^{-2 \alpha h_{1 M}} \int_{t-h_{1 M}}^{t} \dot{z}^{T}(s) R \dot{z}(s) d s \leq-e^{-2 \alpha h_{1 M}}\left[z^{T}(t) R z(t)\right. \\
& -2 z^{T}(t) R z\left(t-h_{1 M}\right) \\
& \left.+z^{T}\left(t-h_{1 M}\right) R z\left(t-h_{1 M}\right)\right] \text {. }
\end{aligned}
$$

Note that

$$
\begin{aligned}
-\delta \int_{t-h_{1 M}}^{t-h_{1 m}} \dot{z}^{T}(s) U \dot{z}(s) d s= & -\left(h_{1 M}-h(t)\right) \int_{t-h_{1 M}}^{t-h(t)} \dot{z}^{T}(s) U \dot{z}(s) d s \\
& -\left(h(t)-h_{1 m}\right) \int_{t-h_{1 M}}^{t-h(t)} \dot{z}^{T}(s) U \dot{z}(s) d s \\
& -\left(h(t)-h_{1 m}\right) \int_{t-h(t)}^{t-h_{1 m}} \dot{z}^{T}(s) U \dot{z}(s) d s \\
& -\left(h_{1 M}-h(t)\right) \int_{t-h(t)}^{t-h_{1 m}} \dot{z}^{T}(s) U \dot{z}(s) d s .
\end{aligned}
$$

Using Lemma 2 and let $\beta=\frac{h_{1 M}-h(t)}{h_{1 M}-h_{1 m}} \leq 1$. Then

$$
\begin{aligned}
& -\delta \int_{t-h_{1 M}}^{t-h_{1 m}} \dot{z}^{T}(s) U \dot{z}(s) d s \\
& \leq-\left[z(t-h(t))-z\left(t-h_{1 M}\right)\right]^{T} U\left[z(t-h(t))-z\left(t-h_{1 M}\right)\right]
\end{aligned}
$$




$$
\begin{aligned}
& -\left[z\left(t-h_{1 m}\right)-z(t-h(t))\right]^{T} U\left[z\left(t-h_{1 m}\right)-z(t-h(t))\right] \\
& -\beta\left[z\left(t-h_{1 m}\right)-z(t-h(t))\right]^{T} U\left[z\left(t-h_{1 m}\right)-z(t-h(t))\right] \\
& -(1-\beta)\left[z(t-h(t))-z\left(t-h_{1 M}\right)\right]^{T} U\left[z(t-h(t))-z\left(t-h_{1 M}\right)\right] .
\end{aligned}
$$

By using the following identity relation

$$
\begin{aligned}
0= & -2 \dot{z}^{T}(t) P \dot{z}(t)-2 \dot{z}^{T}(t) A P z(t)+2 \dot{z}^{T}(t) C f(e(t)) \\
& +2 \dot{z}^{T}(t) D g\left(e\left(t-h_{1}(t)\right)\right)+2 \dot{z}^{T}(t) E \int_{t-k_{1}(t)}^{t} h(e(s)) d s \\
& -2 \dot{z}^{T}(t) B_{1} Y z(t)+2 \dot{z}^{T}(t) B_{2}(t) u\left(t-h_{2}(t)\right) \\
& +2 \dot{z}^{T}(t) B_{3} \int_{t-k_{2}(t)}^{t} u(s) d s .
\end{aligned}
$$

By using Lemma 1 and 2, we have

$$
\begin{aligned}
2 \dot{z}^{T}(t) C f(e(t)) \leq & \dot{z}^{T}(t) C^{T} U_{1} C \dot{z}(t) \\
& +z^{T}(t) P F^{T} U_{1}^{-1} F P z(t), \\
2 \dot{z}^{T}(t) D g\left(e\left(t-h_{1}(t)\right)\right) \leq & \dot{z}^{T}(t) D^{T} U_{2} D \dot{z}(t) \\
& +z^{T}\left(t-h_{1}(t)\right) P G^{T} U_{2}^{-1} G P z\left(t-h_{1}(t)\right), \\
2 \dot{z}^{T}(t) E \int_{t-k_{1}(t)}^{t} h(e(s)) d s \leq \quad & 2 k_{1} e^{2 \alpha k_{1}} \dot{z}^{T}(t) E^{T} U_{3} E \dot{z}(t) \\
& +\frac{e^{-2 \alpha k_{1}}}{2} \int_{t-k_{1}(t)}^{t} h^{T}(e(s)) U_{3}^{-1} h(e(s)) d s, \\
2 \dot{z}^{T}(t) B_{2}(t) u\left(t-h_{2}(t)\right) \leq & 3 e^{2 \alpha h_{2} \dot{z}^{T}(t) B_{2}^{T} S_{1} B_{2} \dot{z}(t)} \\
& +\frac{e^{-2 \alpha h_{2}}}{3} u^{T}\left(t-h_{2}(t)\right) S_{1}^{-1} u\left(t-h_{2}(t)\right), \\
2 \dot{z}^{T}(t) B_{3} \int_{t-k_{2}(t)}^{t} u(s) d s \leq & 2 k_{2} e^{2 \alpha k_{2}} \dot{z}^{T}(t) B_{3}^{T} S_{2} B_{3} \dot{z}(t) \\
& +\frac{e^{-2 \alpha k_{2}}}{2} \int_{t-k_{2}(t)}^{t} u^{T}(s) S_{2}^{-1} u(s) d s,
\end{aligned}
$$

From (20) - (27), we obtain

$$
\begin{aligned}
\dot{V}(e(t))+2 \alpha V(e(t)) \leq & \xi^{T}(t)\left((1-\beta) \mathcal{M}_{1}+\beta \mathcal{M}_{2}\right) \xi(t) \\
& +z^{T}(t) \mathcal{M}_{3} z(t)+\dot{z}^{T}(t) \mathcal{M}_{4} \dot{z}(t) \\
& +z^{T}\left(t-h_{1}(t)\right) \mathcal{M}_{5} z\left(t-h_{1}(t)\right)
\end{aligned}
$$


where

$$
\begin{aligned}
\mathcal{M}_{3}= & -0.1 R\left(e^{-2 \alpha h_{1 m}}+e^{-2 \alpha h_{1 M}}\right) \\
& +2 P F^{T} U_{1}^{-1} F P+k_{1} P H^{T} U_{3}^{-1} H P+2 e^{-2 \alpha h_{2}} Y^{T} S_{1}^{-1} Y \\
\mathcal{M}_{4}= & -0.1 P+h_{2}^{2} Y^{T} S_{1}^{-1} Y \\
\mathcal{M}_{5}= & -0.1 e^{-2 \alpha h_{1 M}} U+2 P G^{T} U_{2}^{-1} G P, \\
\mathcal{M}_{6}= & Q_{1}+P^{-1} Y^{T} Q_{2} Y P^{-1} \\
\xi(t)= & {\left[z(t), \dot{z}(t), z\left(t-h_{1 m}\right), z(t-h(t)), z\left(t-h_{1 M}\right)\right] . }
\end{aligned}
$$

Since $0 \leq \beta \leq 1,(1-\beta) \mathcal{M}_{1}+\beta \mathcal{M}_{2}$ is a convex combination of $\mathcal{M}_{1}$ and $\mathcal{M}_{2}$. Therefore, $(1-\beta) \mathcal{M}_{1}+\beta \mathcal{M}_{2}<0$ is equivalent to $\Gamma_{1}<0$ and $\Gamma_{2}<0$. Applying Schur complement lemma, the inequalities $\mathcal{M}_{3}<0, \mathcal{M}_{4}<0$ and $\mathcal{M}_{5}<0$ are equivalent to $\Gamma_{3}<0, \Gamma_{4}<0$ and $\Gamma_{5}<0$, respectively. Thus, it follows from (8) - (12) and (28), we obtain

$$
\dot{V}(e(t))+2 \alpha V(e(t)) \leq 0, \quad \text { for } n \omega \leq t \leq n \omega+\delta .
$$

Thus, by the above differential inequality (29), we have

$$
\dot{V}(e(t)) \leq V(e(n \omega)) e^{-2 \alpha t-n \omega}, \quad \text { for } n \omega \leq t \leq n \omega+\delta .
$$

Case II: for $n \omega+\delta \leq t \leq(n+1) \omega$, we choose Lyapunov-Krasovskii functional having the following form :

$$
V(e(t))=\sum_{i=1}^{7} V_{i}
$$

where $V_{i}(t), 1=1,2, \ldots, 7$ are defined similar in (18). We are able to do similar estimation as we did for Case I, we have the following

$$
\begin{aligned}
\dot{V}(e(t))+2(\alpha-\varepsilon) V(e(t)) \leq & \xi^{T}(t)\left((1-\beta) \mathcal{N}_{1}+\beta \mathcal{N}_{2}\right) \xi(t) \\
& +z^{T}(t) \mathcal{N}_{3} z(t) \\
& +z^{T}\left(t-h_{1}(t)\right) \mathcal{N}_{4} z\left(t-h_{1}(t)\right),
\end{aligned}
$$

where

$$
\begin{aligned}
\mathcal{N}_{3}= & -0.1 R\left(e^{-2 \alpha h_{1 m}}+e^{-2 \alpha h_{1 M}}\right) \\
& +2 P F^{T} U_{1}^{-1} F P+k_{1} P H^{T} U_{3}^{-1} H P, \\
\mathcal{N}_{4}= & \mathcal{M}_{4} .
\end{aligned}
$$


Since $0 \leq \beta \leq 1,(1-\beta) \mathcal{N}_{1}+\beta \mathcal{N}_{2}$ is a convex combination of $\mathcal{N}_{1}$ and $\mathcal{N}_{2}$. Therefore, $(1-\beta) \mathcal{N}_{1}+\beta \mathcal{N}_{2}<0$ is equivalent to $\Gamma_{6}<0$ and $\Gamma_{7}<0$. Applying Schur complement lemma, the inequalities $\mathcal{N}_{3}<0, \mathcal{N}_{4}<0$ are equivalent to $\Gamma_{8}<0, \Gamma_{5}<0$, respectively. Thus, it follows from (12) - (15) and (32), we obtain

$$
\dot{V}(e(t))-2(\varepsilon-\alpha) V(e(t)) \leq 0, \quad \text { for } n \omega \leq t \leq n \omega+\delta .
$$

Thus, by the above differential inequality (29), we have

$$
\dot{V}(e(t)) \leq V(e(n \omega+\delta)) e^{2(\varepsilon-\alpha)(t-n \omega-\delta)}, \quad \text { for } n \omega \leq t \leq n \omega+\delta .
$$

By (30) and (34), we have

$$
\begin{aligned}
V(e((n+1) \omega)) \leq & V(e(n \omega+\delta)) e^{2(\varepsilon-\alpha)(\omega-\delta)} \\
\leq & V(e(n \omega)) e^{-2 \alpha \delta} e^{2(\varepsilon-\alpha)(\omega-\delta)} \\
= & V(e(n \omega)) e^{-2 \alpha \delta+2(\varepsilon-\alpha)(\omega-\delta)} \\
\leq & V(e((n-1) \omega+\delta)) e^{2 \rho(\omega-\delta)} e^{-2 \alpha \delta+2(\varepsilon-\alpha)(\omega-\delta)} \\
\leq & V(e((n-1) \omega)) e^{-2 \alpha \delta+2(\varepsilon-\alpha)(\omega-\delta)} e^{-2 \alpha \delta+2(\varepsilon-\alpha)(\omega-\delta)} \\
= & V(e((n-1) \omega)) e^{2(-2 \alpha \delta+2(\varepsilon-\alpha)(\omega-\delta))} \\
& \vdots \\
\leq & V(e(0)) e^{(-2 \alpha \delta+2(\varepsilon-\alpha)(\omega-\delta))(n+1)} .
\end{aligned}
$$

For any $t>0$, there is a $n_{0} \geq 0$, such that $n_{0} \omega \leq t \leq\left(n_{0}+1\right) \omega$.

Case 1. For $n_{0} \omega+\delta \leq t \leq\left(n_{0}+1\right) \omega$, using condition (16), we have

$$
\begin{aligned}
V(e(t)) & \leq V\left(e\left(n_{0} \omega+\delta\right)\right) e^{2(\varepsilon-\alpha)\left(t-\left(n_{0} \omega+\delta\right)\right)} \\
& \leq V\left(e\left(n_{0} \omega\right)\right) e^{-2 \alpha \delta} e^{2(\varepsilon-\alpha)\left(t-\left(n_{0} \omega+\delta\right)\right)} \\
& \leq V(e(0)) e^{(-2 \alpha \delta+2(\varepsilon-\alpha)(\omega-\delta)) n_{0}} e^{-2 \alpha \delta} e^{2(\varepsilon-\alpha)\left(t-\left(n_{0} \omega+\delta\right)\right)} \\
& \leq V(e(0)) e^{(-2 \alpha \delta+2(\varepsilon-\alpha)(\omega-\delta)) n_{0}} e^{-2 \alpha \delta} e^{2(\varepsilon-\alpha)\left(\left(n_{0}+1\right) \omega-\left(n_{0} \omega+\delta\right)\right)} \\
& =V(e(0)) e^{(-2 \alpha \delta+2(\varepsilon-\alpha)(\omega-\delta))\left(n_{0}+1\right)} \\
& =V(e(0)) e^{\frac{(-2 \alpha \delta+2(\varepsilon-\alpha)(\omega-\delta))\left(n_{0}+1\right) \omega}{\omega}} \\
& \leq V(e(0)) e^{\frac{(-2 \alpha \delta+2(\varepsilon-\alpha)(\omega-\delta)) t}{\omega}}
\end{aligned}
$$

Case 2. For $n_{0} \omega \leq t \leq n_{0} \omega+\delta$, using condition (16), we have

$$
\begin{aligned}
V(e(t)) & \leq V\left(e\left(n_{0} \omega\right)\right) e^{-2 \alpha\left(t-n_{0} \omega\right)} \\
& \leq V(e(0)) e^{(-2 \alpha \delta+2(\varepsilon-\alpha)(\omega-\delta)) n_{0}} e^{-2 \alpha\left(t-n_{0} \omega\right)}
\end{aligned}
$$




$$
\begin{aligned}
& \leq V(e(0)) e^{(-2 \alpha \delta+2(\varepsilon-\alpha)(\omega-\delta)) n_{0}} \\
& =V(e(0)) e^{-(-2 \alpha \delta+2(\varepsilon-\alpha)(\omega-\delta))} e^{(-2 \alpha \delta+2(\varepsilon-\alpha)(\omega-\delta))\left(n_{0}+1\right)} \\
& =V(e(0)) e^{-(-2 \alpha \delta+2(\varepsilon-\alpha)(\omega-\delta))} e^{\frac{(-2 \alpha \delta+2(\varepsilon-\alpha)(\omega-\delta))\left(n_{0}+1\right) \omega}{\omega}} \\
& \leq V(e(0)) e^{-(-2 \alpha \delta+2(\varepsilon-\alpha)(\omega-\delta))} e^{\frac{(-2 \alpha \delta+2(\varepsilon-\alpha)(\omega-\delta)) t}{\omega}} .
\end{aligned}
$$

Let $\xi=e^{-(-2 \alpha \delta+2(\varepsilon-\alpha)(\omega-\delta))}$. By (35) and (36), we have

$$
V(e(t)) \leq \xi V(e(0)) e^{\frac{(-2 \alpha \delta+2(\varepsilon-\alpha)(\omega-\delta)) t}{\omega}}, \quad \forall t \geq 0
$$

On the other hand, using the condition (19), we have obtained the following:

$$
\|e(t)\| \leq \sqrt{\frac{V(e(0)) \xi}{\gamma}} e^{\frac{(-\alpha \delta+(\varepsilon-\alpha)(\omega-\delta)) t}{\omega}}, \quad \forall t \geq 0
$$

which implies the error system (5) is exponentially stable under the controller $H 1$, then the controlled slave system (1) is synchronized with the master system (2). The proof is thus completed.

\section{Numerical Examples}

In this section, we now provide an example to show the effectiveness of the result in Theorem 2 .

Example 4.1 Consider the cellular neural networks with various activation functions and mixed time-varying delays using hybrid intermittent feedback control with the following parameters :

$$
\begin{aligned}
\dot{x}(t)= & -A x(t)+C \tilde{f}(x(t))+D \tilde{g}\left(x\left(t-h_{1}(t)\right)\right) \\
& +E \int_{t-k_{1}(t)}^{t} \tilde{h}(x(s)) d s+I(t), \\
x(t)= & \phi_{1}(t), \quad t \in[-d, 0], \\
\dot{y}(t)= & -A y(t)+C \tilde{f}(y(t))+D \tilde{g}\left(y\left(t-h_{1}(t)\right)\right) \\
& +E \int_{t-k_{1}(t)}^{t} \tilde{h}(y(s)) d s+I(t)+\mathcal{U}(t) \\
y(t)= & \phi_{2}(t), \quad t \in[-d, 0],
\end{aligned}
$$


where

$$
\begin{aligned}
A & =\left[\begin{array}{ll}
1 & 0 \\
0 & 1
\end{array}\right], C=\left[\begin{array}{cc}
0.3 & -0.2 \\
0.1 & -0.3
\end{array}\right], D=\left[\begin{array}{cc}
0.8 & 0.4 \\
-0.3 & 0.5
\end{array}\right], \\
E & =\left[\begin{array}{cc}
0.5 & 0.2 \\
-0.3 & 0.5
\end{array}\right], F=\left[\begin{array}{cc}
0.4 & 0 \\
0 & 0.2
\end{array}\right], G=\left[\begin{array}{cc}
0.3 & 0 \\
0 & 0.2
\end{array}\right], \\
H & =\left[\begin{array}{cc}
0.2 & 0 \\
0 & 0.3
\end{array}\right], B_{1}=\left[\begin{array}{ll}
4 & 0 \\
0 & 1
\end{array}\right], B_{2}=\left[\begin{array}{ll}
3 & 0 \\
0 & 1
\end{array}\right], \\
B_{3} & =\left[\begin{array}{ll}
2 & 0 \\
0 & 1
\end{array}\right], \\
\phi_{1}(t) & =[-0.4 \cos t, 0.5 \cos t], \quad \phi_{2}(t)=[\sin t, \sin t] .
\end{aligned}
$$

Solution: From the conditions (8)-(15) of Theorem 2, we let $\alpha=0.07, \varepsilon=0.09$, $\omega=4, \delta=2.5 h_{1 m}=0.1, h_{1 M}=0.2, h_{2}=0.3, k_{1}=0.15, k_{2}=0.2$. By using the LMI Toolbox in MATLAB, we obtain

$$
\begin{aligned}
P & =\left[\begin{array}{ll}
1.5072 & 0.0147 \\
0.0147 & 0.8106
\end{array}\right], \quad Q=\left[\begin{array}{ll}
0.3204 & 0.0027 \\
0.0027 & 0.1821
\end{array}\right], \\
R & =\left[\begin{array}{ll}
1.5954 & 0.0073 \\
0.0073 & 0.9829
\end{array}\right], \quad U=\left[\begin{array}{ll}
6.5228 & 0.1067 \\
0.1067 & 3.2384
\end{array}\right], \\
S_{1} & =10^{-3}\left[\begin{array}{cc}
0.0446 & -0.4554 \\
-0.4554 & 1.2455
\end{array}\right], \quad S_{2}=\left[\begin{array}{cc}
0.0003 & -0.0017 \\
-0.0017 & 0.0311
\end{array}\right], \\
U_{1} & =\left[\begin{array}{cc}
2.6046 & 0 \\
0 & 0.5215
\end{array}\right], \quad U_{2}=\left[\begin{array}{cc}
0.6454 & 0 \\
0 & 0.1738
\end{array}\right], \\
U_{3} & =\left[\begin{array}{cc}
0.4273 & 0 \\
0 & 0.1726
\end{array}\right], \quad Y=10^{-3}\left[\begin{array}{cc}
0.0362 & 0.0994 \\
0.3908 & 2.7357
\end{array}\right], \\
K & =10^{-3}\left[\begin{array}{cc}
-0.0252 & 0.1231 \\
0.2923 & -3.3803
\end{array}\right] .
\end{aligned}
$$

We let $h_{1}(t)=0.1+0.1|\sin t|, h_{2}(t)=0.3 e^{|\sin t|}, k_{1}(t)=0.15|\cos t|, k_{2}(t)=$ $0.2 e^{|\cos t|}, \phi_{1}(t)=[-0.4 \cos t, 0.5 \cos t], \phi_{2}(t)=[\sin t, \sin t], \forall t \in[-0.3,0]$ and the activation function as follows:

$$
\begin{aligned}
f_{1}\left(x_{1}(t)\right) & =0.2\left(\left|x_{1}(t)+1\right|-\left|x_{1}(t)-1\right|\right), \\
f_{2}\left(x_{2}(t)\right) & =0.1\left(\left|x_{1}(t)+1\right|-\left|x_{1}(t)-1\right|\right), \\
g_{1}\left(x_{1}(t)\right) & =0.15\left(\left|x_{1}(t)+1\right|-\left|x_{1}(t)-1\right|\right), \\
g_{2}\left(x_{2}(t)\right) & =0.1\left(\left|x_{2}(t)+1\right|-\left|x_{2}(t)-1\right|\right), \\
h_{1}\left(x_{1}(s)\right) & =0.1 \tanh \left(-4 x_{1}(s)\right),
\end{aligned}
$$




$$
h_{2}\left(x_{2}(s)\right)=0.15 \tanh \left(5 x_{2}(s)\right) .
$$

Figure 1. shows the trajectories of solutions $e_{1}(t)$ and $e_{2}(t)$ of the cellular neural networks with various activation functions and mixed time-varying delays without hybrid intermittent feedback control $(\mathcal{U}(t)=0)$. Figure 2. shows the trajectories of solutions $e_{1}(t)$ and $e_{2}(t)$ of the the cellular neural networks with various activation functions and mixed time-varying delays with hybrid intermittent feedback control

$$
\mathcal{U}(t)=\left\{\begin{array}{c}
-10^{-3}\left[\begin{array}{cc}
-0.1010 & 0.4924 \\
0.2923 & -3.3803
\end{array}\right] e(t) \\
-10^{-3}\left[\begin{array}{cc}
-0.0757 & 0.3693 \\
0.2923 & -3.3803
\end{array}\right] e\left(t-h_{2}(t)\right) \\
-10^{-3}\left[\begin{array}{cc}
-0.0505 & 0.2462 \\
0.2923 & -3.3803
\end{array}\right] \int_{t-k_{2}(t)}^{t} e(s) d s \\
n \omega \leq t \leq n \omega+\delta, \\
0, n \omega+\delta<t \leq(n+1) \omega .
\end{array}\right.
$$

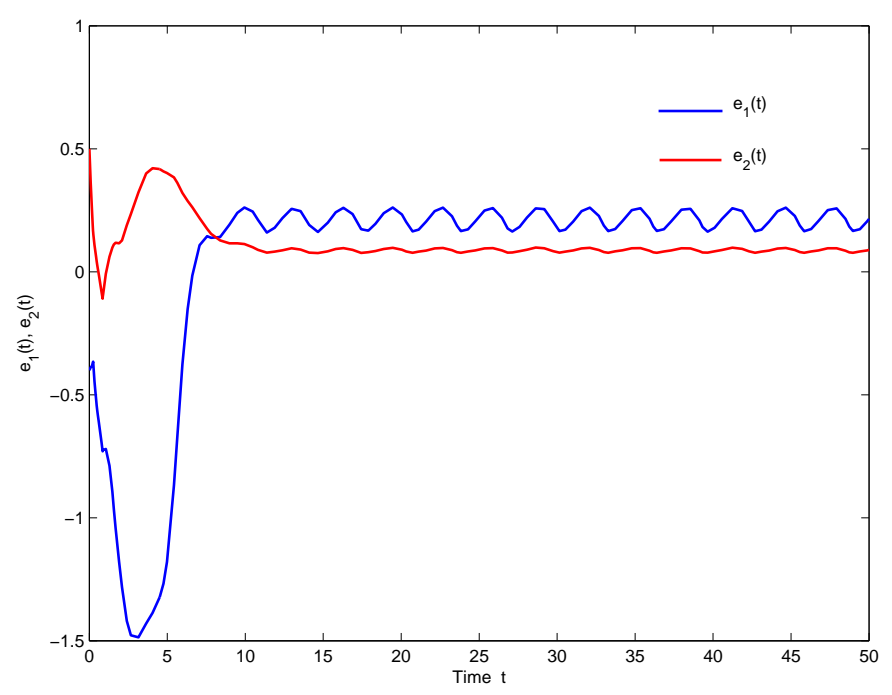

Figure 1: shows the trajectories of solutions $e_{1}(t)$ and $e_{2}(t)$ of the cellular neural networks without hybrid intermittent feedback control $(\mathcal{U}(t)=0)$. 


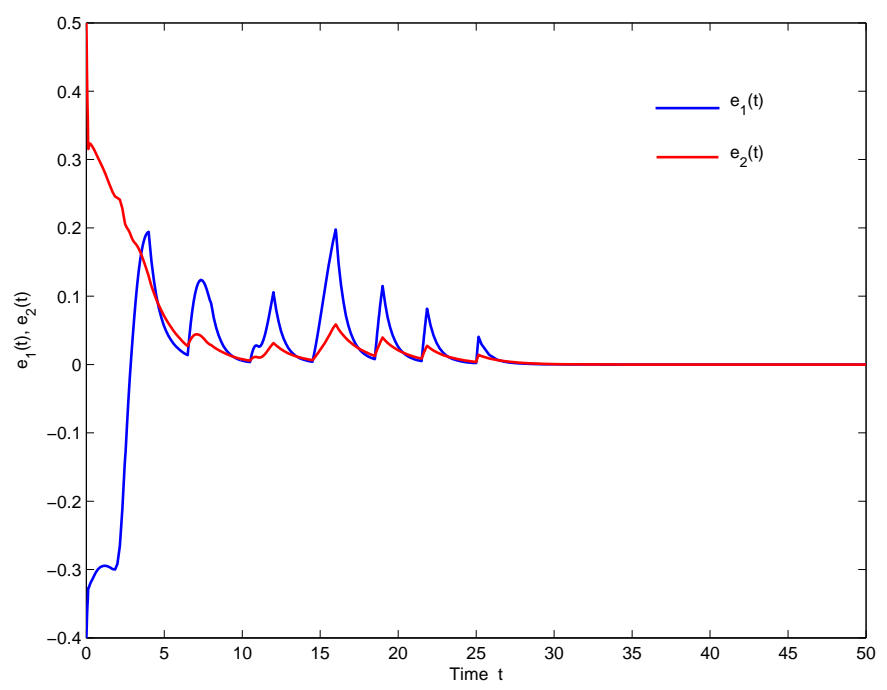

Figure 2: shows the trajectories of solutions $e_{1}(t)$ and $e_{2}(t)$ of the cellular neural networks with hybrid intermittent feedback control (39).

\section{Conclusions}

In this paper, we have investigated the exponential synchronization of cellular neural networks with various activation functions and mixed time-varying delays via hybrid intermittent feedback control. The interval time-varying delay function is not necessary to be differentiable which allows time-delay function to be a fast time-varying function. A new class of Lyapunov-Krasovskii functional is constructed to new delay-dependent sufficient conditions for the exponential synchronization of the error systems have been derived by a set of LMIs without introducing any free-weighting matrices. The hybrid intermittent feedback controller designed can guarantee exponential stability of the error system. Simulation results have been given to illustrate the effectiveness of the proposed method.

\section{Acknowledgments}

We would like to thank referees for their valuable comments and suggestions. This work is supported by the Thailand Research Fund(TRF), the Office of the Higher Education Commission(OHEC), Srinakharinwirot University (grant 
number MRG5580081). We would like to thank Assoc. Prof. Dr. Piyapong Niamsup for valuable comments and suggestions.

\section{References}

[1] M. M. Gupta, L. Jin and N. Homma, Static and Dynamic Neural Networks: From Fundamentals to Advanced Theory, New York: Wiley, 2003.

[2] L. M. Pecora and T. L. Carroll, Synchronization in chaotic systems, Phys. Rev. Lett., 64 (1990), 821-824.

[3] J. Liang and J. Cao, Global asymptotic stability of bi-directional associative memory networks with distributed delays, Appl. Math. Comput., 152 (2004), 415-424.

[4] H. Zhao, Global asymptotic stability of Hopfield neural network involving distributed delays, Neural Netw., 17 (2004), 47-53.

[5] M. V. Thuan, Guaranteed cost control of neural networks with various activation functions and mixed time-varying delays in state and control, Differential equations and control processes, 3 (2011), 18-29.

[6] V. N. Phat and H. Trinh, Exponential stabilization of neural networks with varous activation functions and mixed time-varying delays, IEEE Trans. Neural Networks, 21 (2010), 1180-1184.

[7] T. Botmart and P. Niamsup, Adaptive control and synchronization perturbed Chuas system, Math. Comput. Simulation, 75 (2007), 37-55.

[8] T. Botmart and W. Weera, Guaranteed Cost Control for Exponential Synchronization of Cellular Neural Networks with Mixed Time-Varying Delays via Hybrid Feedback Control, Abstr. Appl. Anal. , 2013 (2013), $1-12$.

[9] T. Botmart, P. Niamsup and X. Liu, Synchronization of non-autonomous chaotic systems with time-varying delay via delayed feedback control, Commun. Nonlinea.r Sci. Numer. Simulat., 17 (2012), 189-1907.

[10] T. Li, S.-M. Fei and K.-J. Zhang, Synchronization control of recurrent neural networks with distributed delays, Physica A, 387 (2008), 982-996. 
[11] Z.-G. Wu, P. Shi, H. Su and J. Chu, Exponential synchronization of neural networks with discrete and distributed delays under time-varying sampling, IEEE Trans. Neural Netw, 23 (2012), 1368-1376.

[12] J. H. Park, On global stability criterion of neural networks eiyh continuously distributed delays, Chaos Solitons Fractals, 37 (2008), 444-449.

[13] Zochowski M, Intermittent dynamical control, Phys D., 145 (2000), 18190.

[14] X. Yang, J. Cao, Stochastic synchronization of coupled neural networks with intermittent control, Phys. Lett. A., 373 (2009), 3359-3272.

[15] W. Zhang, J. Huang, P. Wei, Weak synchronization of chaotic neural networks with parameter mismatch via periodically intermittent control, Appl. Math. Model., 35 (2011), 612-620.

[16] J. Yu, C. Hu, H. Jiang, Z. Teng, Exponential synchronization of CohenGrossberg neural networks via periodically intermittent control, Neurocomputing, 74 (2011), 1776-1782.

[17] H. Zhu, B. Cui, Stabilization and synchronization of chaotic systems via intermittent control, Commun Nonlinear Sci Numer Simulat., 15 (2010), 3577-3586.

[18] G. Zhang, X. Lin, X. Zhang, Exponential Stabilization of Neutral-Type Neural Networks with Mixed Interval Time-Varying Delays by Intermittent Control, Circuits Systems Signal Process., 33 (2014), 371-391.

[19] K. Gu, V.L. Kharitonov and J.Chen, Stability of time-delay system, Boston: Birkhauser; 2003. 